\title{
A dynamic model of the frequency-dependent rupture process of the 2011 Tohoku-Oki earthquake
}

\author{
Yihe Huang, Lingsen Meng, and Jean-Paul Ampuero \\ Division of Geological and Planetary Sciences, California Institute of Technology, Pasadena, CA 91125, U.S.A.
}

(Received December 27, 2011; Revised May 7, 2012; Accepted May 17, 2012; Online published January 28, 2013)

\begin{abstract}
We present a 2D dynamic rupture model that provides a physical interpretation of the key features of the 2011 Tohoku-Oki earthquake rupture. This minimalistic model assumes linear slip-weakening friction, the presence of deep asperities and depth-dependent initial stresses. It reproduces the first-order observations of the alongdip rupture process during its initial $100 \mathrm{~s}$, such as large static slip and low-frequency radiation up-dip from the hypocenter, and slow rupture punctuated by high-frequency radiation in deeper regions. We also derive quantitative constraints on the ratio of shallow versus deep radiation from teleseismic back-projection source imaging. This ratio is explained in our model by the rupture of deep asperities surrounded by low stress drop regions, and by the decrease of initial stresses towards the trench.
\end{abstract}

Key words: Dynamic rupture model, Tohoku earthquake, back projection, high-frequency radiation, deep asperities.

\section{Introduction}

The data recorded during the $M_{\mathrm{w}} 9.02011$ Tohoku-Oki earthquake provides a transformative opportunity to gain insight into the physics of devastating mega-earthquakes. Numerous studies are shaping our view of the static and kinematic aspects of the rupture process of this event. A striking feature is the large slip in the shallower region. Oceanbottom pressure gauge, tsunami and multi-beam bathymetric data indicate that slip near the trench is as large as 50 to $80 \mathrm{~m}$ (Fujiwara et al., 2011; Ito et al., 2011; Kanamori and Yomogida, 2011). Kinematic slip inversions also place the area of largest slip up-dip from the hypocenter (Kanamori and Yomogida, 2011; Koketsu et al., 2011; Pollitz et al., 2011). Another striking feature is the distinct location of high-frequency (HF) and low-frequency (LF) slip. Teleseismic back-projection studies find that the HF energy around $1 \mathrm{~Hz}$ originates from the deeper region (Kanamori and Yomogida, 2011; Meng et al., 2011; Yao et al., 2011), while other studies point to the low-frequency radiation coming from the shallower region (Simons et al., 2011; Wei et al., 2012). The present work aims to provide a dynamic interpretation of the contrasted frequency content of slip in the deeper and shallower regions of the Tohoku-Oki earthquake rupture. To quantitatively constrain the dynamic model, we derive in Section 2 an estimate of the ratio of shallow versus deep HF source amplitude from the back-projection source imaging of Meng et al. (2011).

The evolution of the rupture speed down-dip from the hypocenter is also constrained by back-projection studies. Meng et al. (2011) described it as a very slow initial

Copyright (C) The Society of Geomagnetism and Earth, Planetary and Space Sciences (SGEPSS); The Seismological Society of Japan; The Volcanological Society of Japan; The Geodetic Society of Japan; The Japanese Society for Planetary Sciences; TERRAPUB.

doi:10.5047/eps.2012.05.011 phase $(<10 \mathrm{~s})$, followed by a stage of regular rupture speed $(<20 \mathrm{~s})$, and finally a stage of slow rupture speed of order 1 $\mathrm{km} / \mathrm{s}$ interspersed with $\mathrm{HF}$ radiation episodes (<90 s). Because the slip rate, rise time and up-dip rupture speed are less robustly determined by current studies, we do not include them as first-order constraints in our model.

In Section 3, we formulate a 2D dynamic rupture model that is consistent with the key observations described above. Simulation results are presented and discussed in Sections 4 and 5. Our model is obviously not unique (Goto and Sawada, 2010) and is deliberately minimal in its assumptions. Its scope is limited to the initial $100 \mathrm{~s}$ of the rupture process, before the rupture became dominated by alongstrike rupture propagation. It nevertheless constitutes a demonstration of a conceptual interpretation of the depthdependent frequency content and slow down-dip rupture of the Tohoku-Oki earthquake proposed by Meng et al. (2011). It also provides a basis for more sophisticated $3 \mathrm{D}$ dynamic modeling (Galvez et al., 2011).

\section{Back Projection Constraints on Shallow vs. Deep HF Source Amplitude}

Meng et al. (2011) employed a novel, high-resolution multitaper-MUSIC array processing technique to image the rupture process of the Tohoku-Oki earthquake at high frequency, around $1 \mathrm{~Hz}$, based on teleseismic data recorded by the USArray and the European seismic network. Location and timing of HF radiation sources were robustly constrained but less attention was paid to their amplitude. In order to provide a quantitative constraint on HF source amplitude, we derive an upper bound on the HF slip rate power in the shallower regions relative to the deeper regions. We estimate the minimum value of the amplitude ratio between shallow secondary peaks over deep primary peaks of the back-projection images that allows the detection of shal- 

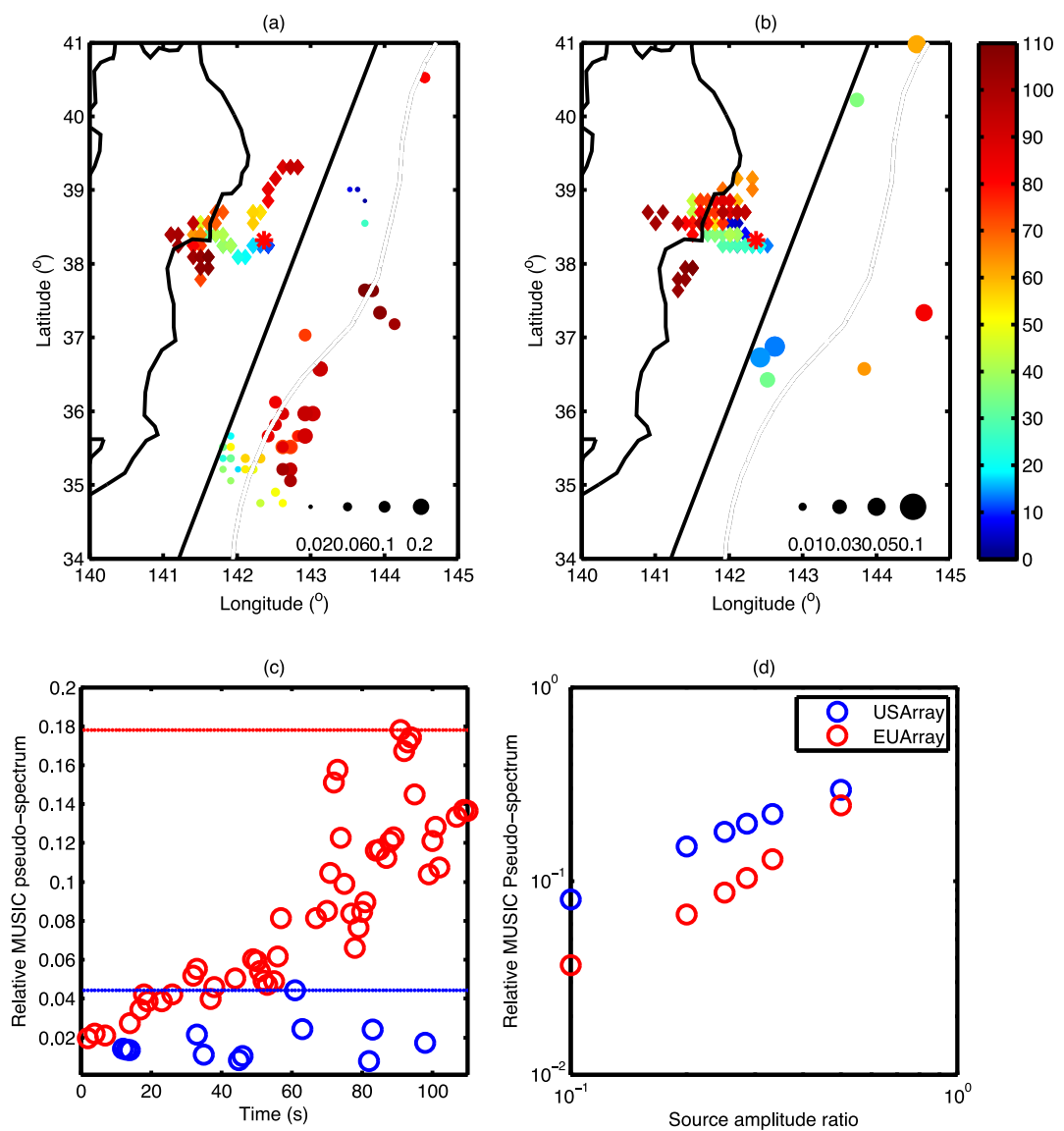

Fig. 1. Shallow secondary features of the Tohoku-Oki earthquake identified in MUSIC back-projection from (a) USArray and (b) European array. The thick black line, double line curve, and the black line east of the epicenter (red star) are the Japanese coastline, the trench and our conventional boundary between "deep" and "shallow" sources, respectively. The deep high-frequency radiators (diamonds) and shallow local maxima (circles) are also shown. The color indicates the rupture time. The size of the circles denotes the ratio between the amplitude of the shallow MUSIC pseudo-spectrum peak and that of the contemporaneous global maximum. (c) The ratio between shallow and deep simultaneous MUSIC pseudo-spectrum maxima for the European array (red) and USArray (blue). The dashed lines indicate the largest ratio for each array. (d) Shallow/deep MUSIC pseudo-spectrum ratio as a function of the shallow/deep source amplitude ratio. (Color figure is in online version.)

low sources without ambiguity. We then convert this into a threshold on HF slip rate amplitude ratios.

We define the boundary between "deep" and "shallow" as a line striking 210 degrees at a distance of 0.5 degrees east from the epicenter. In each frame of the first $110 \mathrm{~s}$ of Meng et al. (2011)'s back-projection images of the USArray and European array data, we identify the largest secondary peak of the MUSIC pseudo-spectrum at shallow depth (Figs. 1(a) and 1(b)). These secondary peaks are of uncertain origin: they can be either true shallow sources, sidelobes of the deep sources or artifacts introduced by coda waves. We compute the ratio of these secondary peaks (when they exist) over the deep global maxima (Fig. 1(c)). We take the maximum ratio over all time frames to represent the threshold for the unambiguous detection of shallow sources: $\sim 0.18$ for the European array and $\sim 0.04$ for the USArray. The larger threshold at the European array is attributed to stronger artifacts and aliasing due to its sparser station distribution, although these artifacts do not affect the analysis of the strongest deep radiators.

To convert this threshold on the MUSIC pseudospectrum ratio into a threshold on relative source amplitude, we analyze synthetic scenarios comprising two simultaneous point sources, located 1-degree down-dip and 1-degree up-dip, respectively, from the JMA mainshock epicenter. The 2-degrees distance is approximately the separation between the location of the HF radiators (Meng et al., 2011) and the peak slip region close to the trench (Fujiwara $e t$ al., 2011; Ito et al., 2011; Koketsu et al., 2011). Synthetic Green's functions at teleseismic distance, including the effect of a regional velocity model for Japan (Miura et al., 2005), were computed by interfacing a spectral element code (SPECFEM3D, Tromp et al., 2008) and a generalized ray theory code (Chu et al., 2009). We applied MUSIC back-projection to the synthetic waveforms from doublesource scenarios with a range of seismic moment ratios between the shallow and deep sources, and measured the ratio of shallow vs. deep MUSIC pseudo-spectrum peaks. We obtained a power-law relationship between the MUSIC pseudo-spectrum ratio and the source amplitude ratio (Fig. 1(d)). Combining these calibration curves with the MUSIC pseudo-spectrum ratio threshold derived previously, we estimate that the source amplitude ratio threshold is 0.5 for the European array and 0.1 for USArray. We conclude that during the Tohoku-Oki earthquake the deep sources were at least 10 times as strong as the shallow ones at $1 \mathrm{~Hz}$. 


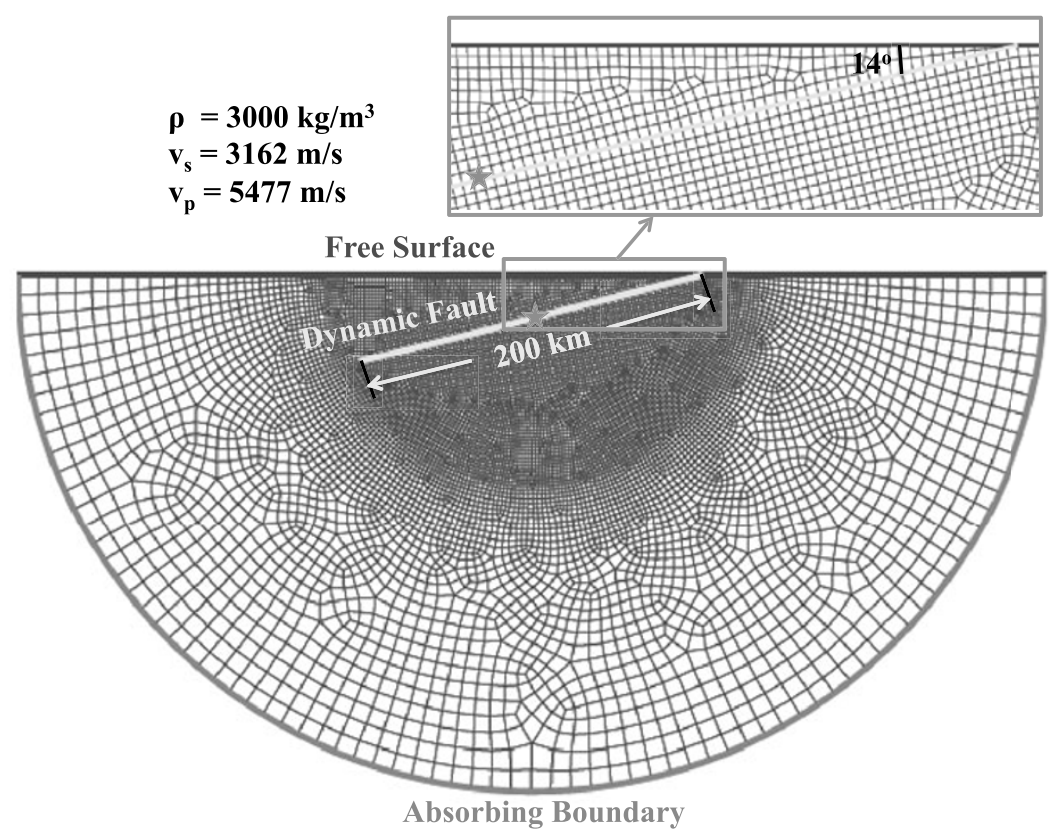

Fig. 2. The unstructured mesh with a free boundary on the top and an absorbing boundary in a semicircle. The hypocenter is in the middle of the 200-km-long fault. The zoom-in picture shows the mesh around the fault and the fault dipping angle. The density, $S$ velocity, and $P$ velocity, are indicated in the top left.

\section{Dynamic Rupture Model Assumptions}

Our 2D dynamic rupture model of the Tohoku-Oki earthquake comprises a $200-\mathrm{km}$-wide thrust fault with a dip angle of $14^{\circ}$ embedded in a homogeneous elastic half space (Fig. 2). The assumed density, shear modulus and Poisson's ratio are $3000 \mathrm{~kg} / \mathrm{m}^{3}, 30 \mathrm{GPa}$ and 0.25 , respectively. The fault is governed by the linear slip-weakening friction law with static and dynamic friction coefficients of 0.6 and 0.2 , respectively, and a critical slip-weakening distance of $3 \mathrm{~m}$, except in five deep asperities in which the critical distance is $1 \mathrm{~m}$. The shallower region of the Tohoku megathrust has usually been considered weakly coupled (Loveless and Meade, 2010), since few earthquakes had occurred there. In comparison, the deeper region has ruptured several times in $M_{\mathrm{w}} 7$ and $M_{\mathrm{w}} 8$ events and hosts numerous repeating earthquake sequences (Igarashi et al., 2003). Thus, the deeper region certainly contains asperities with a range of sizes (Kanamori and Yomogida, 2011), while the shallower region might be dominated by a large asperity with a much longer recurrence time.

Furthermore, the effective normal stress on the subducted plate interface should increase with depth, as a result of the overburden pressure, and decrease significantly in areas of large pore fluid pressure. Tobin and Saffer (2009) found an extremely low effective stress in the Nankai subduction zone from the trench to a down-dip distance of $20 \mathrm{~km}$ due to elevated pore pressures. Though similar studies are still lacking in Tohoku, we found that such a variation of effective normal stress can explain the rupture behavior of the shallower region. Based on the observation on the Nankai subduction zone, the effective normal stress is set to $10 \mathrm{MPa}$ in a 20-km-wide region near the trench and then increases linearly up to $100 \mathrm{MPa}$ at $80 \mathrm{~km}$ from the trench.

Figure 3(a) shows our assumed distributions of initial shear stress, static strength and dynamic strength along the fault. The hypocenter is surrounded by a large asperity with high initial shear stress. The shear stress is then reduced in the shallower region and tapered in accordance with the effective normal stress. In the deeper region, we set five small asperities surrounded by areas with negative stress drop. The spontaneous dynamic rupture problem is solved with the 2D spectral element code SEM2DPACK (http://www.sourceforge.net/projects/sem2d/, see e.g. Huang and Ampuero, 2011). The mesh is unstructured and includes progressive coarsening towards a circular absorbing boundary $300 \mathrm{~km}$ away from the epicenter (Fig. 2). We nucleate the rupture using the time-weakening procedure of Andrews (1985) with an imposed rupture speed of $800 \mathrm{~m} / \mathrm{s}$. The rupture starts propagating spontaneously after $15 \mathrm{~s}$.

\section{Numerical Results}

We show in Fig. 3(b) the spatio-temporal distribution of slip rate resulting from our simulation. The rupture propagates bilaterally from the hypocenter. Outside the nucleation region, the up-dip rupture accelerates and reaches the Rayleigh wave speed $(\sim 2.9 \mathrm{~km} / \mathrm{s})$. Near the trench a supershear rupture front emerges ahead of the main front, accompanied by an acceleration of slip rate up to $\sim 6.5 \mathrm{~m} / \mathrm{s}$ due to the effect of the free surface (Nielsen, 1998). A shallow weak layer, velocity-strengthening friction, or off-fault dissipation, can help inhibit the supershear transition near the free surface (Kaneko and Lapusta, 2010). After the rupture reaches the trench, a strong slip acceleration front is generated by the reflection from the free surface (see e.g. figure 5a of Ma and Beroza, 2008). The front modulates the slip along the fault again, and the final slip in the shallower region is between 64 and $74 \mathrm{~m}$ (Fig. 3(c)).

In the down-dip direction, the rupture speeds up to $\sim 2$ $\mathrm{km} / \mathrm{s}$ before $\sim 25 \mathrm{~s}$, and then decelerates to $\sim 1 \mathrm{~km} / \mathrm{s}$ after reaching the region of small asperities, which radiate high- 

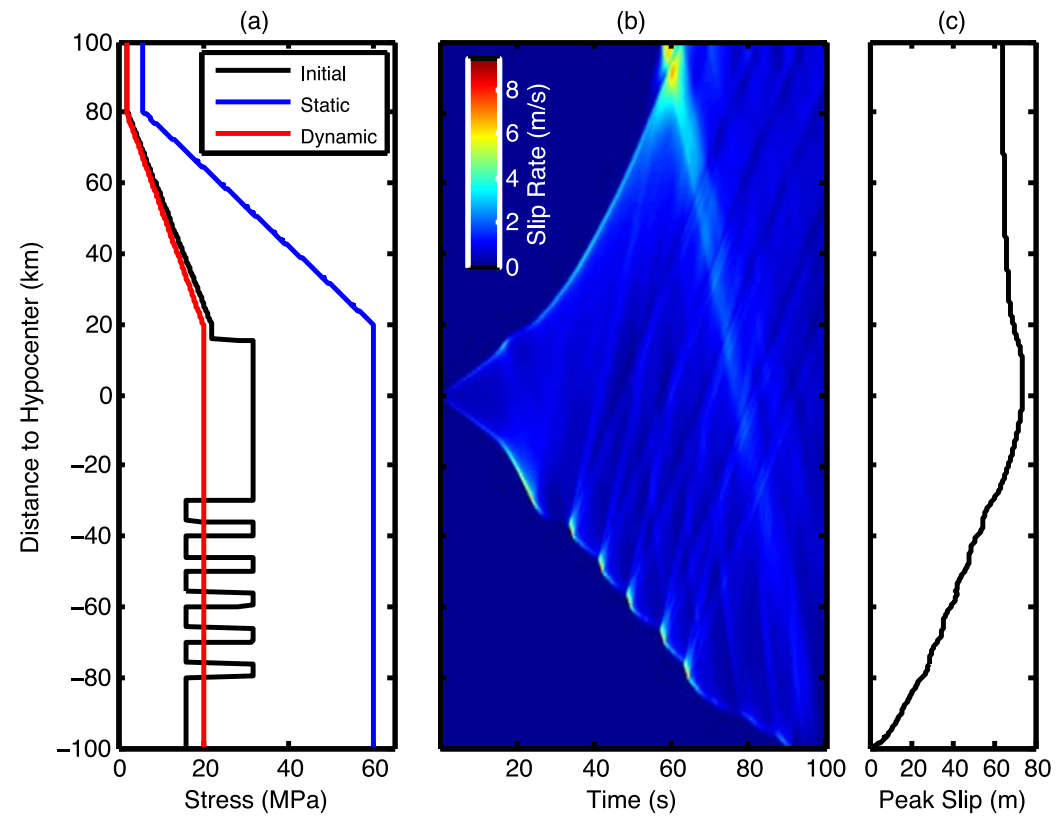

Fig. 3. (a) Initial shear stress, static strength and dynamic strength in the numerical model set-up as a function of distance to the hypocenter. (b) Spatiotemporal distribution of slip rate. (c) Peak slip as a function of distance to the hypocenter. (Color figure is in online version.)

(a)

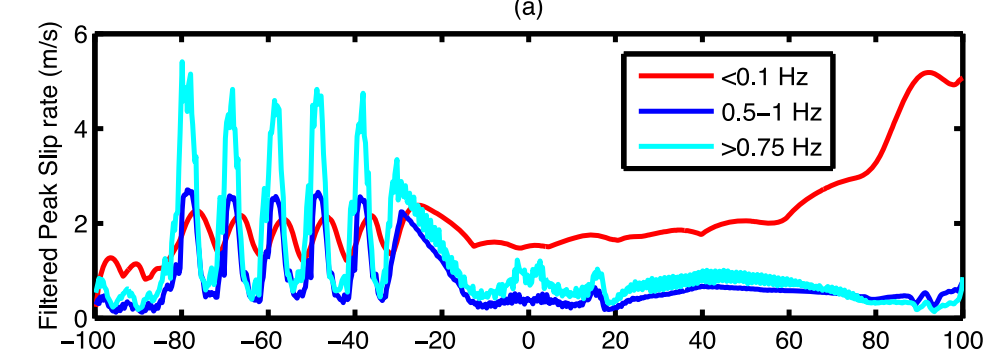

(b)

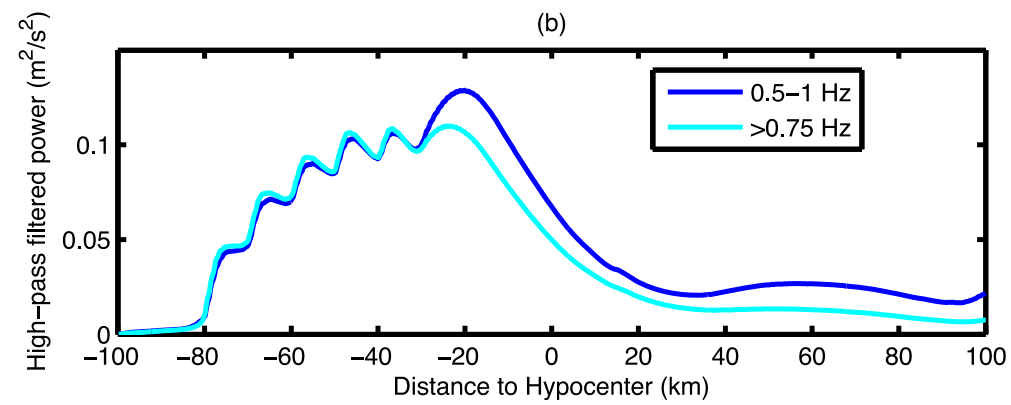

Fig. 4. (a) Low-pass filtered $(<0.1 \mathrm{~Hz})$ and high-pass filtered $(0.5-1.0 \mathrm{~Hz}$ and $>0.75 \mathrm{~Hz})$ peak slip rates as a function of distance to the hypocenter. (b) Calculated power of the high-pass filtered peak slip rates. (Color figure is in online version.)

frequency energy. The fast down-dip propagation followed by slow propagation with high-frequency bursts is a key feature in the back-propagation results of Meng et al. (2011). The strong $P$ and $S$ phases generated by each small asperity interfere with each other and produce an interesting pattern in the spatio-temporal distribution of slip rate (Fig. 3(b)). The peak slip rate inside the small asperities is around 9 $\mathrm{m} / \mathrm{s}$. However, this does not lead to a large slip at depth because of the overall small stress drop. The final slip tapers almost linearly towards the bottom of the seismogenic zone (Fig. 3(c)).

To study the spatial complementarity between LF and HF slip, we filter the simulated slip rate in different frequency bands: an LF band with frequencies lower than $0.1 \mathrm{~Hz}$, an $\mathrm{HF}$ band with frequencies of $0.5-1 \mathrm{~Hz}$, and an HF band with frequencies higher than $0.75 \mathrm{~Hz}$. We plot the $\mathrm{HF}$ and LF peak slip rate values in Fig. 4(a). The $0.5-1 \mathrm{~Hz}$ peak slip rate in the deeper region is about 4 times as large as that in the shallower region, and it is roughly 5 times for frequencies higher than $0.75 \mathrm{~Hz}$. Simulations that ignore the tapering of initial stresses towards the trench produce a very large HF slip near the trench, while simulations without deep asperities reproduce the observed slow front but not the deep HF radiation.

To facilitate the comparison between our simulation and the back-projection result, we compute the HF slip-rate 
power over a $10 \mathrm{~s}$ sliding windows and apply a spatial Gaussian smoothing with a half-width of $50 \mathrm{~km}$, comparable to the main lobe width of the array response of the USArray and the European array (Fig. 4(b)). We find that the overall source power in the deeper region is at least 3 times larger than in the shallower region, and the power ratio increases with frequency. Hence, the simulation demonstrates that a depth-dependent distribution of asperities is a viable mechanism to explain why the Tohoku-Oki earthquake radiated more HF energy from its deeper regions.

\section{Conclusions and Discussion}

We present a 2D dynamic rupture simulation that incorporates multiple deep asperities and a depth-dependent initial stress. The simulation successfully reproduces the following first-order observations of the rupture process of the 2011 Tohoku-Oki earthquake: a large portion of the final slip in the shallower region, the spatial separation between sources of low-frequency and high-frequency slip, and a period of slow down-dip rupture propagation punctuated by high-frequency bursts. We develop an estimate of the ratio of slip-rate power in the shallower versus the deeper regions, based on the MUSIC back projection analysis, which we then reproduce in our dynamic rupture simulations.

Our results indicate that the stress state is very different between the shallower and deeper regions. Though the initial stress in the shallower region is barely above the dynamic strength, the reflected waves inside the front wedge induce a high transient stress drop and large final slip, but no significant overshoot is found. Based on the consistency of our result and the observations, we infer that the initial stress in the shallower region is very low compared to the static strength. Thus, it would be difficult to nucleate an earthquake in the shallower region, but the final slip will always be amplified once the rupture propagates through. This behavior should naturally exist in shallow subduction events. A lower initial stress (e.g. equal to the dynamic strength) still allows the rupture to break up to the trench, but leads to a small final slip $(<50 \mathrm{~m})$ in the shallower region.

In the deeper region, the stress state inside and outside the asperities is also distinct. To reproduce the slow average down-dip rupture speed, the initial stress needs to be lower than the dynamic strength in the regions between the asperities. These regions conceptually represent the places where overshoot occurred in the previous earthquakes. Hence, though stress accumulated in the interseismic period, the initial stress still lies below the dynamic strength, which acted as a barrier and lowered the rupture speed during the Tohoku-Oki earthquake. We have explored a range of models of the deep fault region defined by the size of asperities, their density (the inverse of their spacing), their stress drop and the background initial stress in between. Slow rupture is favored by low values of these parameters, but too low values lead to rupture arrest. A large HF radiation requires large values of those parameters, but too large values lead to fast rupture and large slip. Hence, the family of viable models is somewhat constrained.

Our assumption of slip-weakening friction leads to a crack-like rupture instead of a pulse-like rupture (Heaton,
1990). It is still difficult to identify if the Tohoku-Oki earthquake is dominated by crack or pulse behavior. Mechanisms for pulse-like rupture, such as thermal pressurization, in faults with heterogeneous mechanical or hydraulic properties (Noda and Lapusta, 2010) might provide an alternative interpretation of the variable frequency content of the slip rate.

The back-propagating slip front emerging from the interaction of dynamic rupture on a dipping fault and the free surface might have contributed to the reactivation of the Tohoku-Oki earthquake rupture at about $100 \mathrm{~s}$. However, the possibly accompanying strong HF radiation near the trench is not observed. In our simulation, this is suppressed by the tapered initial stresses towards the trench. Alternatively, this might also be achieved by velocity-strengthening or velocity-neutral friction, but at the expense of reducing too much final slip near the trench. Other mechanisms left for future simulations are the anelastic deformation of the frontal wedge and dynamically triggered slip on splay faults (Kanamori and Yomogida, 2011).

Acknowledgments. This work was supported by NSF grants EAR-0944288 and EAR-1015704, the Gordon and Betty Moore Foundation and SCEC (funded by NSF EAR-0106924 and USGS 02HQAG0008 cooperative agreements). We thank Wenbo Wu for generating the Green's functions for our synthetic tests, and Surendra Somala for generating the meshes for our dynamic rupture simulations.

\section{References}

Andrews, D. J., Dynamic plane-strain shear rupture with a slip-weakening friction law calculated by a boundary integral method, Bull. Seismol. Soc. Am., 75(1), 1-21, 1985.

Chu, R., L. P. Zhu, and D. V. Helmberger, Determination of earthquake focal depths and source time functions in central Asia using teleseismic $\mathrm{P}$ waveforms, Geophys. Res. Lett., 36, doi:10.1029/2009gl039494, 2009.

Fujiwara, T., S. Kodaira, T. No, Y. Kaiho, N. Tkahashi, and Y. Kaneda, The 2011 Tohoku-Oki earthquake: Displacement reaching the trench axis, Science, 334, 1240, doi:10.1126/science.1211554, 2011.

Galvez, P., J.-P. Ampuero, L. A. Dalguer, and T. Nissen-Meyer, Dynamic rupture modeling of the $2011 \mathrm{M} 9$ Tohoku earthquake with an unstructured 3D spectral element method, AGU Fall Meet. Suppl., Abstract U51B-0043, 2011.

Goto, H. and S. Sawada, Trade-offs among dynamic parameters inferred from results of dynamic source inversion, Bull. Seismol. Soc. Am., 100(3), 910-922, doi:10.1785/0120080250, 2010.

Heaton, T. H., Evidence for and implications of self-healing pulses of slip in earthquake rupture, Phys. Earth Planet. Inter, 64(1), 1-20, 1990.

Huang, Y. and J.-P. Ampuero, Pulse-like rupture induced by low-velocity fault zones, J. Geophys. Res., 116, B12307, doi:10.1029/20011JB008684, 2011.

Igarashi, T., T. Matsuzawa, and A. Hasegawa, Repeating earthquakes and interplate aseismic slip in the northeastern Japan subduction zone, $J$. Geophys. Res., 108(B5), 2249, doi:10.1029/2002JB001920, 2003.

Ito, Y., T. Tsuji, Y. Osada, M. Kido, D. Inazu, Y. Hayashi, H. Tsushima, R. Hino, and H. Fujimoto, Frontal wedge deformation near the source region of the 2011 Tohoku-Oki earthquake, Geophys. Res. Lett., 38, L00G05, doi:10.1029/2011GL048355, 2011.

Kanamori, H. and K. Yomogida, Preface. First Results of the 2011 Off the Pacific Coast of Tohoku Earthquake, Earth Planets Space, 63(7), 511, doi:10.5047/eps.2011.07.019, 2011.

Kaneko, Y. and N. Lapusta, Supershear transition due to a free surface in 3-D simulations of spontaneous dynamic rupture on vertical strike-slip faults, Tectonophysics, 493, 272-284, 2010.

Koketsu, K. et al., A unified source model for the 2011 Tohoku earthquake, Earth Planet. Sci. Lett., 210, 480-487, doi:10.1016/j.eps1.2011.09.09, 2011.

Loveless, J. P. and B. J. Meade, Geodetic image of plate motions, slip rates, and partitioning of deformation in Japan, J. Geophys. Res., 115, 
B02410, doi:10.1029/2008JB006248, 2010.

Ma, S. and G. C. Beroza, Rupture dynamics on a bimaterial interface for dipping faults, Bull. Seismol. Soc. Am., 98(4), 1642-1658, doi:10.1785/0120070201, 2008.

Meng, L., A. Inbal, and J.-P. Ampuero, A window into the complexity of the dynamic rupture of the $2011 \mathrm{M}_{\mathrm{w}} 9$ Tohoku-Oki earthquake, Geophys. Res. Lett., 38, L00G07, doi:10.1029/2011GL048118, 2011.

Miura, S., N. Takahashi, A. Nakanishi, T. Tsuru, S. Kodaira, and Y. Kaneda, Structural characteristics off Miyagi forearc region, the Japan Trench seismogenic zone, deduced from a wide-angle reflection and refraction study, Tectonophysics, 407, 165-188, 2005.

Nielsen, S. B., Free surface effects on the propagation of dynamic rupture, Geophys. Res. Lett., 25(1), 125-128, 1998.

Noda, H. and N. Lapusta, Three-dimensional earthquake sequence simulations with evolving temperature and pore pressure due to shear heating: Effect of heterogeneous hydraulic diffusivity, J. Geophys. Res., 115, B12314, doi:10.1029/2010JB007780, 2010.

Pollitz, F. F., R. Burgmann, and P. Banerjee, Geodetic slip model of 2011 M9.0 Tohoku earthquake, Geophys. Res. Lett., 38, L00G08, doi:10.1029/2011GL048632, 2011.
Simons, M. et al., The 2011 magnitude 9.0 Tohoku-Oki earthquake: Mosaicking the megathrust from seconds to centuries, Science, 332, 1421, doi:10.1126/science.1206731, 2011.

Tobin, H. J. and D. M. Saffer, Elevated fluid pressure and extreme mechanical weakness of a plate boundary thrust, Nankai Trough subduction zone, Geology, 37(8), 679-682, doi:10.1130/G25752A.1, 2009.

Tromp, J., D. Komatitsch, and Q. Y. Liu, Spectral-element and adjoint methods in seismology, Commun. Comput. Phys., 3(1), 1-32, 2008.

Wei, S., R. Graves, D. Helmberger, J.-P. Avouac, and J. Jiang, Sources of shaking and flooding during the Tohoku-Oki Earthquake: A mixture of rupture styles, Earth Planet. Sci. Lett., doi:10.1016/j.eps1.2012.04.006, 2012.

Yao, H., P. Gerstoft, P. M. Shearer, and C. Mecklenbräuker, Compressive sensing of the Tohoku-Oki Mw 9.0 earthquake: Frequencydependent rupture modes, Geophys. Res. Lett., 38, L20310, doi:10.1029/2011GL049223, 2011.

Y. Huang (e-mail: yihe@gps.caltech.edu), L. Meng, and J.-P. Ampuero 